\title{
AB INITIO STUDY OF SILVER NANOPARTICLES, GRAIN BOUNDARIES AND THEIR QUADRUPLE JUNCTIONS
}

\author{
1,2Svatava POLSTEROVÁ, ${ }^{1,2}$ Monika VŠIANSKÁ, ${ }^{1}$ Martin FRIÁK, ${ }^{1}$ Naděžda PIZÚROVÁ, \\ ${ }^{3}$ Sergey SOKOVNIN, ${ }^{2,1}$ Mojmír ŠOB \\ ${ }^{1}$ Institute of Physics of Materials, v.v.i., Czech Academy of Sciences, Brno, Czech Republic, EU, \\ polsterova@mail.muni.cz, 230038@mail.muni.cz, friak@ipm.cz, pizurova@ipm.cz \\ ${ }^{2}$ Department of Chemistry, Faculty of Science, Masaryk University, Brno, Czech Republic, EU, \\ mojmir@ipm.cz \\ ${ }^{3}$ Institute of Electrophysics Ural Branch RAS, Yekaterinburg, Russia \\ ${ }^{4}$ Ural Federal University, Yekaterinburg, Russia, sokovnin@iep.uran.ru
}

https://doi.org/10.37904/nanocon.2021.4365

\begin{abstract}
Motivated by our experimental research related to silver nanoparticles with various morphologies, we have employed quantum-mechanical calculations to provide our experiments with theoretical insight. We have computed properties of a 181-atom decahedral silver nanoparticle and two types of internal extended defects, $\Sigma 5(210)$ grain boundaries (GBs) and quadruple junctions (QJs) of these GBs. We have employed a supercell approach with periodic boundary conditions. Regarding the thermodynamic stability of the decahedral nanoparticle, its energy is higher than that of a defect-free face-centered cubic (fcc) Ag by $0.34 \mathrm{eV} / \mathrm{atom}$. As far as the $\Sigma 5(210)$ GB is concerned, its energy amounts to $0.7 \mathrm{~J} / \mathrm{m}^{2}$ and we predict that the studied GBs would locally expand the volume of the lattice. Importantly, the system with GBs is found rather close to the limit of mechanical stability. In particular, the computed value of the shear-related elastic constant $\mathrm{C}_{66}$ is as low as 9.4 $\mathrm{GPa}$ with the zero/negative value representing a mechanically unstable system. We thus predict that the $\Sigma 5(210)$ GBs may be prone to failure due to specific shearing deformation modes. The studied GBs have also the value of Poisson's ratio for some loading directions close to zero. Next, we compare our results related solely to $\Sigma 5(210)$ GBs with those of a system where multiple intersecting $\Sigma 5(210)$ GBs form a network of quadruple junctions. The value of the critical elastic constant $\mathrm{C}_{66}$ is higher in this case, $13 \mathrm{GPa}$, and the mechanical stability is, therefore, better in the system with QJs.
\end{abstract}

Keywords: Nanoparticles, silver, grain boundaries, junctions, quantum-mechanical calculations, elasticity

\section{INTRODUCTION}

The dependence of the biological activity of nanoparticles on their morphology is well known, see, e.g., Ref. [1]. The key aspect is the number of $\{111\}$ surface facets that exhibit the highest atomic density as well as the highest electronic charge density [2]. The nanoparticles containing the highest number of $\{111\}$ facets are formed with decahedral or icosahedral shapes, described as 5 or 20 tetrahedra joined by twin boundaries. Because the tetrahedra do not fill the space completely, grain boundaries or elastic strains are required to complete the nanoparticle shape [3]. The nanoparticle morphologies described above are known as multiplytwinned nanoparticles (MTPs) with fivefold symmetry axes. Our experimental research focused on the method of synthesis and morphological analysis. We used the spark erosion method described in Berkowitz et al. [4] to prepare silver nanoparticles. Morphological analyzes were performed using a JEM2100F transmission electron microscope. We received various nanoparticle morphologies (Figure 1), including typical fivefold 
MTPs. Inspired by these results, we performed quantum mechanical calculations to evaluate the stability of nanoparticle morphology containing quadruple junctions (QJs).

First, we have determined the internal atomic structure and the energy of a 181 -atom decahedral nanoparticle of silver. Second, we have used the calculations to characterize the properties of other internal extended defects, in particular, the $\Sigma 5(210)$ GBs as well as their quadruple junctions (QJs), building upon the expertise related to our previous work [5].
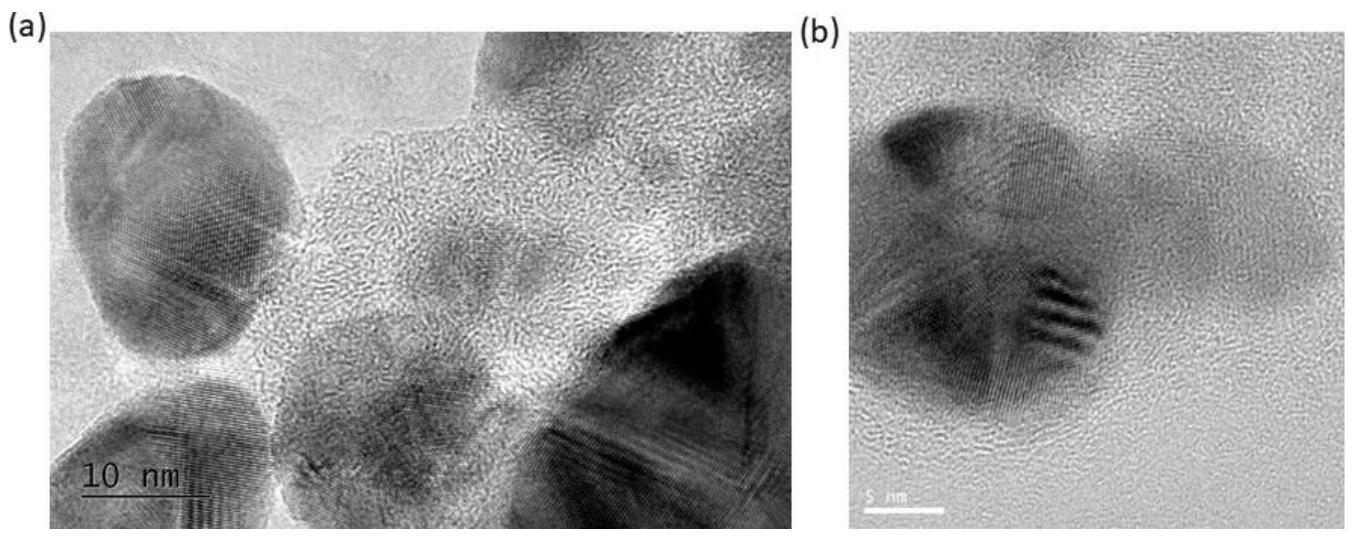

Figure 1 Transmission electron microscopy (TEM) images of Ag nanoparticles illustrating their morphologies (a), especially fivefold MTPs (b)

\section{COMPUTATIONAL METHODOLOGY}

When modeling decahedral silver nanoparticles as well as GBs and their junctions, we have employed a supercell approach. The decahedral nanoparticle is shown in Figure 2. It contains $181 \mathrm{Ag}$ atoms and it was surrounded by a vacuum within a bigger supercell so as to eliminate spurious interaction between an individual nanoparticle and its periodic images in the neighboring cells generated by the periodic boundary conditions. The nanoparticle contains both internal grain boundaries and their fivefold-symmetry junction. Further, supercells modeling $\Sigma 5(210)$ grain boundaries as well as their quadruple junctions (QJs) are shown in Figure 3. The application of periodic boundary conditions in the case of these supercells results in two GBs per supercell visualized in Figure 3a and eight GBs together with four QJs per 180-atom supercell in Figure 3b.

(a)

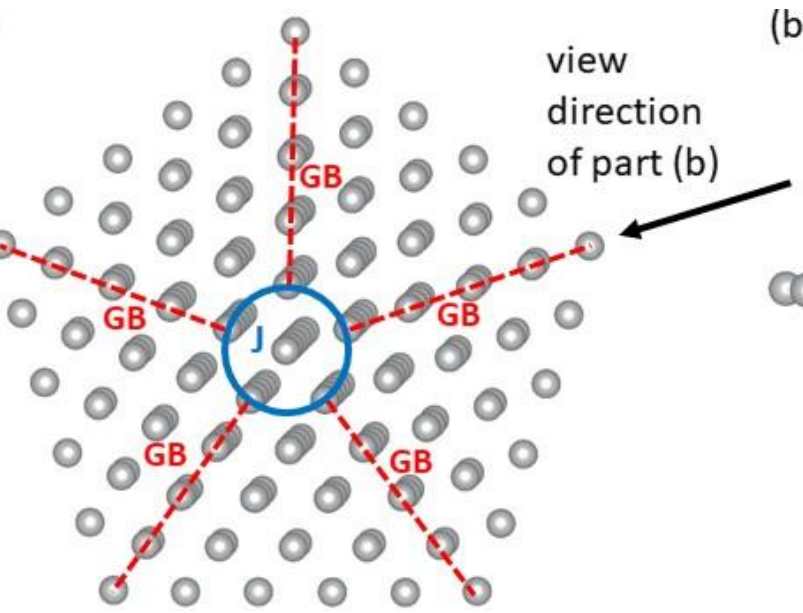

(b) $\quad$ J

J

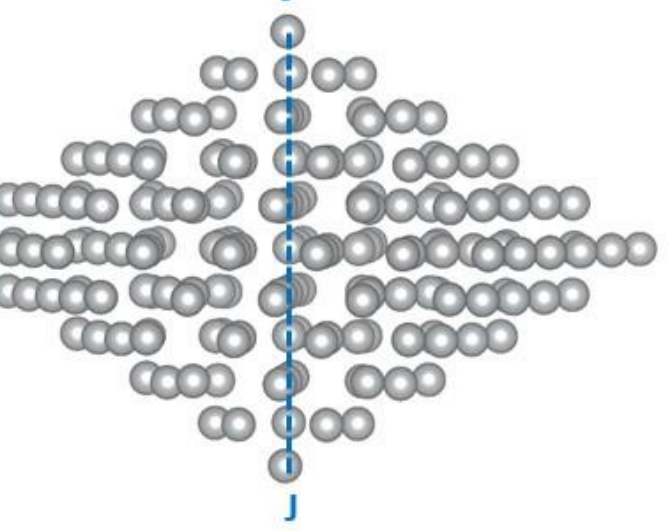

Figure 2 Schematic visualizations of our 181-atom computational model of a decahedral Ag nanoparticle (a) together with its side-view (b). The nanoparticle contains grain boundaries (GBs), see red dashed lines, and junctions (see $\mathrm{J}$ letters) of GBs indicated by blue circles in part (a) and a blue dashed line in part (b). 

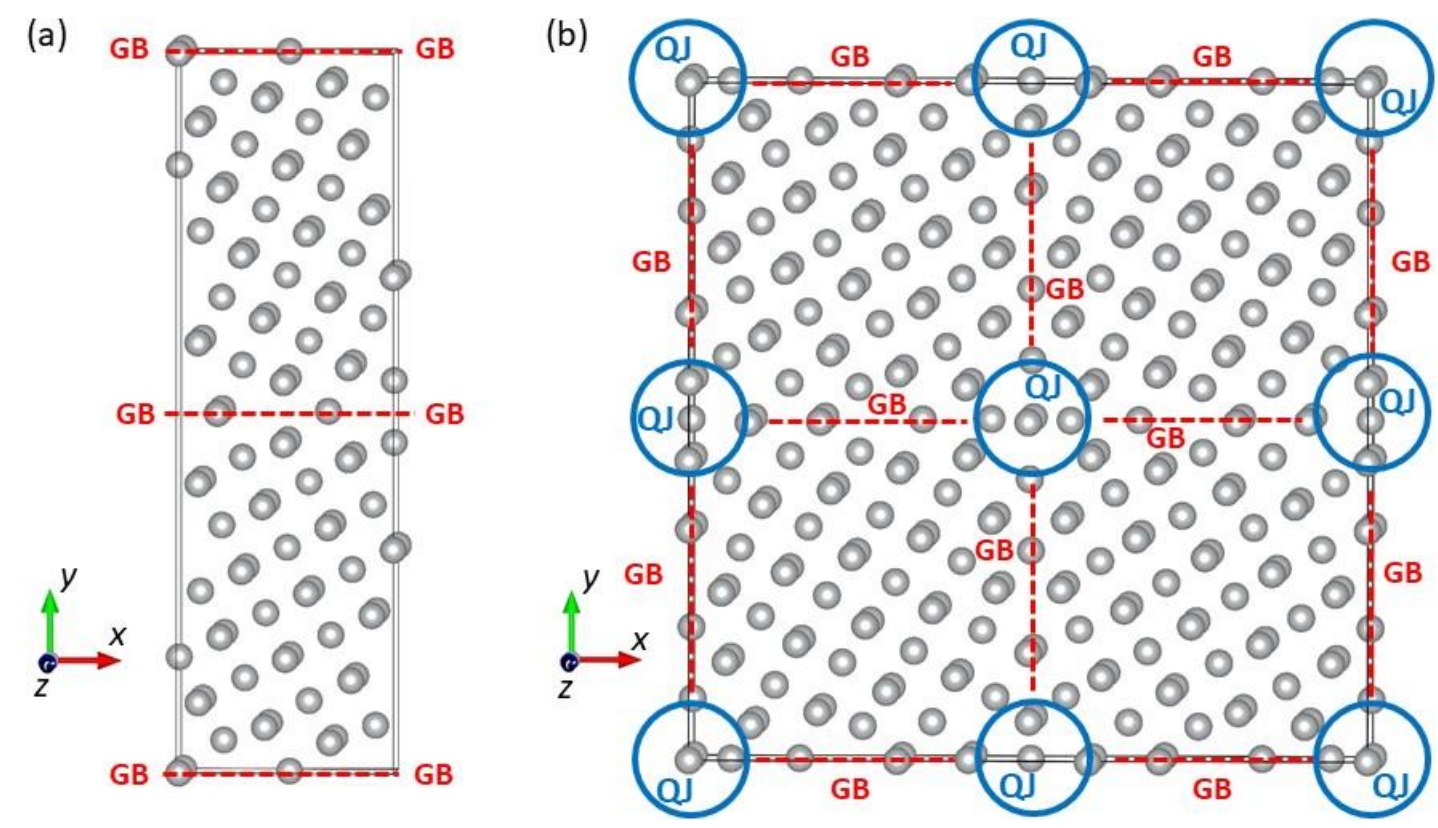

Figure 3 Schematic figures of 64-atom computational supercell modeling $\Sigma 5(210)$ grain boundary (GB) (a) and a 180-atom supercell utilized in our calculations of quadruple junctions (QJ) of $\Sigma 5(210)$ GBs (b). The GB planes are shown as red dashed lines while the regions of quadruple junctions are indicated by blue circles.

Our quantum-mechanical calculations were performed employing the Vienna Ab initio Simulation Package (VASP) [6,7] within the framework of density functional theory [8,9] using projector-augmented-wave (PAW) pseudopotentials $[10,11]$. The exchange and correlation energy were treated in the generalized gradient approximation (GGA) as parametrized by Perdew, Burke and Erhzerhof (PBE'96) [12]. The plane-wave energy cut-off was equal to $450 \mathrm{eV}$ and different Monkhorst-Pack k-point meshes were used for sampling the Brillouin zone in the reciprocal space. In particular, the $3 \times 3 \times 4 \mathrm{k}$-point mesh was applied in the case of the supercell with the lattice parameters $33.8 \AA \times 32.6 \AA \times 24.5 \AA$ containing the 181 -atom decahedral nanoparticle. When computing the $\Sigma 5(210) \mathrm{GB}$, a $6 \times 2 \times 14$ k-point mesh was utilized and $2 \times 2 \times 12 \mathrm{k}$-points were used for 180 -atom supercell modeling QJs of $\Sigma 5(210)$ GBs. Lastly, we applied a $14 \times 14 \times 14$ k-point mesh for calculations of a defect-free face-centered cubic (fcc) elemental silver modeled by a cubic-shape 4-atom cell.

\section{RESULTS}

The 181-atom decahedral nanoparticle has an internally distorted atomic structure as seen in Figure 2. Its energy is higher than that of a defect-free fcc Ag by $0.34 \mathrm{eV} /$ atom. This increase in the energy is caused by the occurrence of a number of extended defects that define the nanoparticle, in particular different surfaces, internal grain boundaries and their central junction, as well as elastic strain field, and further mutual interactions of these defects. It is rather difficult to decompose the energy increase into these individual components. Instead, we use our calculations to assess properties of selected extended defects, $\Sigma 5(210)$ grain boundaries and their junctions, in order to test their thermodynamic and mechanical stability. We start with $\Sigma 5(210)$ GBs that we model with the help of the 64-atom supercell shown in Figure 3a. In order to compute the energy increase due to the studied GBs, we evaluate the GB interface energy. In order to do so, we determine the difference between the energy of 64 -atom supercell and that of 64 atoms of the defect-free fcc Ag. The GB interface energy is then equal to this difference divided by twice the area of the GB interface (because there are two GBs per supercell in Figure 3a). The $\Sigma 5(210)$ GB interface energy amounts to $0.7 \mathrm{~J} / \mathrm{m}^{2}$ (or $0.04 \mathrm{eV} / \AA^{2}$ ). Regarding the structure, the $\Sigma 5(210)$ GB expands locally the volume of the lattice by $2.4 \%$. 
(a)

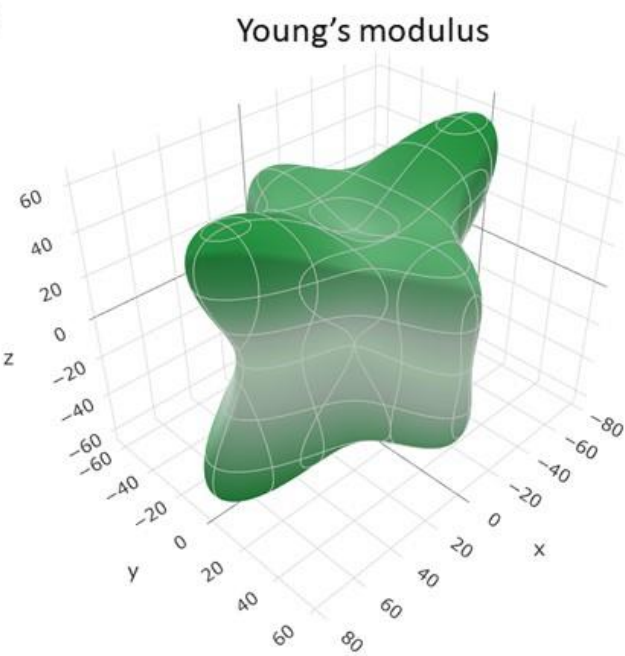

(c)

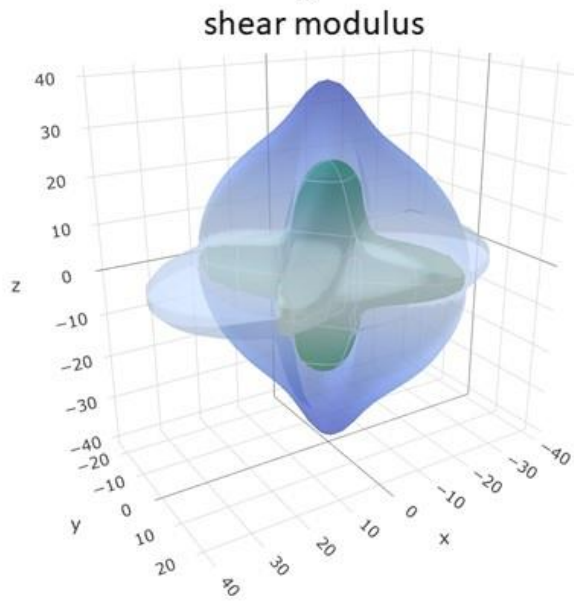

(b) linear compressibility

(d)

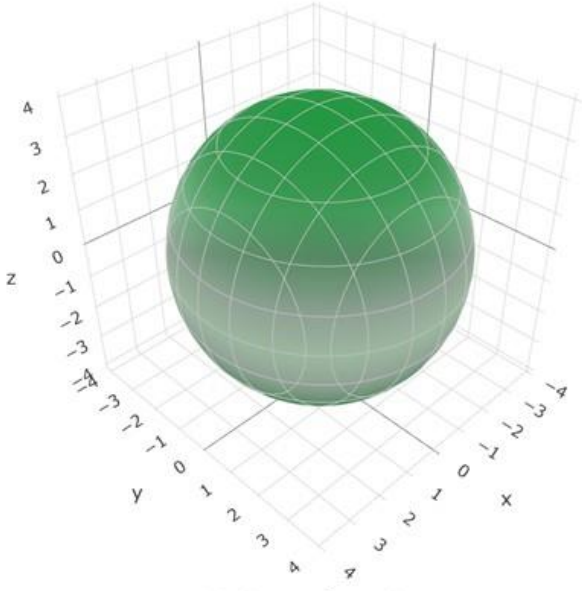

Poisson's ratio

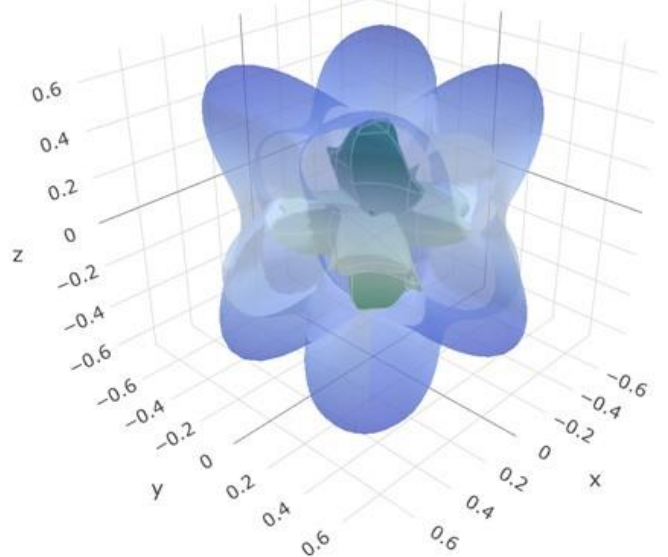

Figure 4 Calculated anisotropic elastic properties of 64 -atom computational supercell modeling the $\Sigma 5(210)$ grain boundary visualized in the form of directional dependences of Young's modulus (a), linear compressi-

bility (b), maximum and minimum values of shear modulus (c) and maximum and minimum values of Poisson's ratio (d). For details see Ref. [13] related to the ELATE software (http://progs.coudert.name/elate).

Next we determine elastic properties of crystalline systems containing the $\Sigma 5(210)$ GBs using the stress-strain method [14]. The elastic properties are characterized by the following set of elastic constants $\mathrm{C}_{11}=119 \mathrm{GPa}$, $\mathrm{C}_{12}=61 \mathrm{GPa}, \mathrm{C}_{13}=79 \mathrm{GPa}, \mathrm{C}_{22}=119 \mathrm{GPa}, \mathrm{C}_{23}=77 \mathrm{GPa}, \mathrm{C}_{33}=102 \mathrm{GPa}, \mathrm{C}_{44}=23 \mathrm{GPa}, \mathrm{C}_{55}=39 \mathrm{GPa}$ and $\mathrm{C}_{66}=9.4 \mathrm{GPa}$. The elasticity is visualized in Figure 4. The Young's modulus is very strongly anisotropic, see Figure 4a, with the minimum and maximum values equal to 29 and $93 \mathrm{GPa}$, respectively. In contrast, the directional dependence of the linear compressibility, see Figure $\mathbf{4 b}$, is very close to a spherical shape with the minimum and maximum values equal to 3.81 and $3.94 \mathrm{TPa}^{-1}$, respectively, i.e. it is nearly isotropic. It is worth noting that systems with a cubic symmetry exhibit perfectly isotropic linear elasticity due to their high symmetry. It is quite interesting that the linear compressibility of the supercell with GBs is so close to the isotropic directional dependence despite of a very low symmetry of its internal configuration of atoms (see Figure 3a). Regarding shear deformations, the maximum and minimum values of shear modulus for different shear directions (for details see Ref. [13]) are shown in Figure 4c. The highest and lowest value out of those for all directions, 39 and $9.4 \mathrm{GPa}$, respectively, are equal to the values of $\mathrm{C}_{55}$ and $\mathrm{C}_{66}$, respectively. If the lowest one would reach zero, the system would become mechanically unstable [15], and the value of $\mathrm{C}_{66}$ is close to this limit. Regarding the maximum and minimum values of Poisson's ratio for different directions shown in Figure $4 \mathbf{d}$, the absolute lowest and highest values are equal to 0.001 and 0.855 , respectively, with the former remarkably close to zero. 
(a)

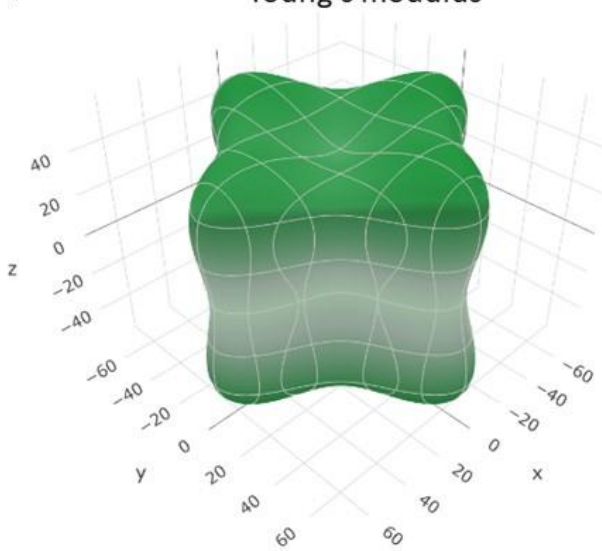

(c)

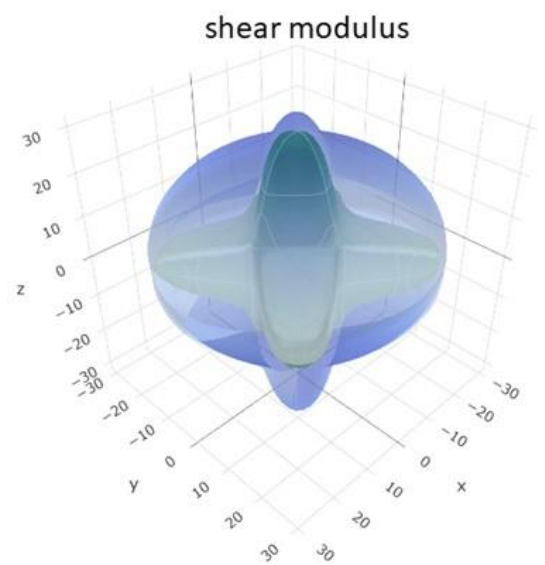

(b) linear compressibility

(d)
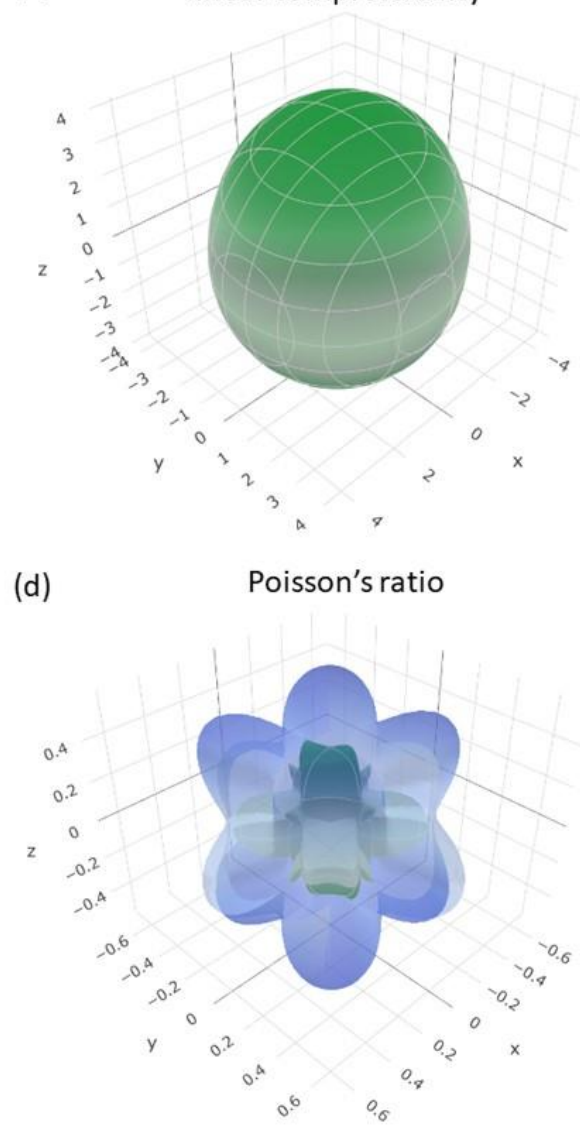

Figure 5 Computed anisotropic elastic properties of 180-atom computational supercell modeling a quadruple junction of four $\Sigma 5(210)$ grain boundaries visualized in the form of directional dependences of Young's modulus (a), linear compressibility (b), maximum and minimum values of shear modulus (c) and maximum and minimum values of Poisson's ratio (d). The figures were obtained using the ELATE software [13].

Regarding the system with quadruple junctions of $\Sigma 5(210) \mathrm{GBs}$, its energy is higher than that of a defect-free fcc Ag by $0.06 \mathrm{eV} /$ atom. It is, unfortunately, difficult to decompose this energy increase into those due to QJs and GBs because it is unclear where exactly the regions associated with GBs and QJs start and finish, see Figure $3 \mathbf{b}$. Therefore, it is not possible to evaluate the corresponding energy contributions. The elastic properties of the system with QJs are characterized by elastic constants $C_{11}=118 \mathrm{GPa}, \mathrm{C}_{12}=56 \mathrm{GPa}, \mathrm{C}_{13}=$ $72 \mathrm{GPa}, \mathrm{C}_{22}=118 \mathrm{GPa}, \mathrm{C}_{23}=74 \mathrm{GPa}, \mathrm{C}_{33}=103 \mathrm{GPa}, \mathrm{C}_{44}=30 \mathrm{GPa}, \mathrm{C}_{55}=30 \mathrm{GPa}$ and $\mathrm{C}_{66}=13 \mathrm{GPa}$. They are visualized in Figure 5. When comparing the elasticity of $\Sigma 5(210) \mathrm{GB}$ in Figure 4 with that of QJ in Figure 5 it is obvious that the Young's modulus, see Figure 5a, is less anisotropic. Its minimum and maximum value equals to 38 and $81 \mathrm{GPa}$, respectively. In contrast, the linear compressibility of the studied QJs is more anisotropic, see Figure $\mathbf{5 b}$, than that of $\Sigma 5(210) \mathrm{GB}$, see Figure $\mathbf{4 b}$, and the minimum and maximum values amount to 3.86 and $4.19 \mathrm{TPa}^{-1}$, respectively. Most of the values of the above listed elastic constants for a system with QJs are very similar to those that we obtained for the system containing solely GBs but the value of the shear-related parameters, see also Figures $\mathbf{4 c}$ and $\mathbf{5 c}$, are different. In particular, the value of $\mathrm{C}_{66}$ is higher (equal to $13 \mathrm{GPa}$ ) in the case of the supercell with QJs when compared with the supercell with GBs $\left(\mathrm{C}_{66}=9.4 \mathrm{GPa}\right)$. It means that the elasticity of the supercell with QJs is further from the limit of the mechanical instability (when $\mathrm{C}_{66}$ would be zero or negative). Regarding the minimum and maximum value of Poisson's ratio (see Figure 5d), the lowest and highest values are equal to 0.050 and 0.699 , respectively. As far as the structure is concerned, the studied system with QJs expands the lattice volume by $3.8 \%$. 


\section{CONCLUSION}

We have performed a series of quantum-mechanical calculations complementing our experimental research focused on silver nanoparticles with various morphologies. In particular, we have computed properties of a 181-atom decahedral silver nanoparticle and two types of internal extended defects, $\Sigma 5(210)$ grain boundaries (GBs) and quadruple junctions (QJs) of these GBs. As far as the thermodynamic stability of the decahedral nanoparticle is concerned, its energy is higher than the energy of a defect-free elemental $\mathrm{Ag}$ by $0.34 \mathrm{eV} /$ atom. Regarding the properties of the $\Sigma 5(210) \mathrm{GB}$, its computed energy is equal to $0.7 \mathrm{~J} / \mathrm{m}^{2}$ and we also predict that the volume of the lattice would be locally expanded due these GBs. Importantly, the system with GBs is predicted to be rather close to the limit of mechanical instability. Specifically, the computed value of the elastic constant $\mathrm{C}_{66}$, that is related to shear deformations, is as low as $9.4 \mathrm{GPa}$ (with the zero or negative values representing a mechanically unstable system). We thus predict that the $\Sigma 5(210) \mathrm{GBs}$ may be prone to a failure due to specific shearing deformation modes. The $\Sigma 5(210)$ GBs have also the lowest values of Poisson's ratio close to zero for some loading directions. Interestingly, when comparing our results related to solely $\Sigma 5(210)$ GBs with those of a system where multiple intersecting $\Sigma 5(210)$ GBs form a network of quadruple junctions, the properties are significantly different. The value of the critical elastic constant $\mathrm{C}_{66}$ is higher, $13 \mathrm{GPa}$, in the system with QJs and its mechanical stability is therefore better. Also the lowest value of Poisson's ratio is not close to zero in the system containing QJs.

\section{ACKNOWLEDGEMENT}

The authors acknowledge the Czech Science Foundation for the financial support received under the Project No. 21-31852J. Computational resources were made available by the Ministry of Education, Youth and Sports of the Czech Republic under the Project of the IT4Innovations National

Supercomputer Center (Project "e-Infrastructure CZ-LM2018140") within the program Projects of Large Research, Development and Innovations Infrastructures and via the CESNET (Project No. LM2015042) and CERIT-Scientific Cloud (Project No. LM20150850). M.F., N.P. and M.Š. acknowledge the institutional support provided by the Czech Academy of Sciences (Project No. UFM-ARVO:68081723). The computational supercells in Figs. 2 and 3 were visualized using the VESTA package [16].

\section{REFERENCES}

[1] WANG, Z. L. (ed.), Characterization of Nanophase Materials, Weinheim, WILEY-VCH Verlag GmbH, 2000.

[2] SONG, M. et al. Influence of morphology and surface exchange reaction on magnetic properties of monodisperse magnetite nanoparticles. Colloids and Surfaces A: Physicochemical and Engineering Aspects. 2012, vol. 408, pp. 114-121.

[3] MARKS, L. D., HOWIE, A. Multiply-twinned particles in silver catalysts. Nature. 1979, vol. 282, pp. $196-198$.

[4] BERKOWITZ A. E., WALTER J. L. Spark erosion: A method for producing rapidly quenched fine powders. Journal of Materials Research. 1987, vol. 2, pp. 277-287.

[5] POLSTEROVÁ, S., FRIÁK, M., VŠIANSKÁ, M., ŠOB, M. Quantum-Mechanical Assessment of the Energetics of Silver Decahedron Nanoparticles, Nanomaterials. 2020, vol. 10, Art. No. 767.

[6] KRESSE, G., HAFNER, J. Ab initio molecular dynamics for liquid metals. Physical Review B. 1996, vol. 47, pp. 558-561.

[7] KRESSE, G., FURTHMŰLLER, J. Efficient iterative schemes for ab initio total-energy calculations using a planewave basis set. Physical Review B. 1996, vol. 54, pp. 11169-11186.

[8] HOHENBERG, P., KOHN, W. Inhomogeneous electron gas. Physical Review B. 1964, vol. 136, pp. B864-B871.

[9] KOHN, W., SHAM, L. J. Self-consistent equations including exchange and correlation effects. Physical Review $A$. 1965, vol. 140, pp. A1133-A1138.

[10] BLŐCHL, P. E. Projector augmented-wave method. Physical Review B. 1994, vol. 50, pp. 17953-17979. 
[11] KRESSE, G., JOUBERT, D. From ultrasoft pseudopotentials to the projector augmented-wave method. Physical Review B. 1999, vol. 59, pp. 1758-1775.

[12] PERDEW, J. P., BURKE, K., ERNZERHOF, M. Generalized gradient approximation made simple. Physical Review Letters. 1996, vol. 77, pp. 3865-3868.

[13] GAILLAC, R.; PULLUMBI, P.; COUDERT, F.X. ELATE: an open-source online application for analysis and visualization of elastic tensors. Journal of Physics: Condensed Matter. 2016, vol. 28, Art. No. 275201.

[14] ZHOU, L.; HOLEC, D.; MAYRHOFER, P.H. First-principles study of elastic properties of cubic Cr1-xAlxN alloys. Journal of Applied Physics. 2013, vol. 113, Art. No. 043511.

[15] MOUHAT, F.; COUDERT, F.X. Necessary and sufficient elastic stability conditions in various crystal systems. Physical Review B. 2014, vol. 90, Art. No. 224104.

[16] MOMMA, K., IZUMI, F. VESTA 3 for three-dimensional visualization of crystal, volumetric and morphology data. Journal of Applied Crystalography. 2011, vol. 44, pp. 1272-1276. 